\title{
Review
}

\section{Activation of the Renin-Angiotensin System and Chronic Hypoxia of the Kidney}

\author{
Masaomi NANGAKU ${ }^{1)}$ and Toshiro FUJITA ${ }^{1)}$
}

\begin{abstract}
Recent studies emphasize the role of chronic hypoxia in the kidney as a final common pathway to end-stage renal failure (ESRD). Hypoxia of tubular cells leads to apoptosis or epithelial-mesenchymal transdifferentiation, which in turn exacerbates the fibrosis of the kidney with the loss of peritubular capillaries and subsequent chronic hypoxia, setting in train a vicious cycle whose end-point is ESRD. While fibrotic kidneys in an advanced stage of renal disease are devoid of peritubular capillary blood supply and oxygenation to the corresponding region, imbalances in vasoactive substances can cause chronic hypoxia even in the early phase of kidney disease. Among various vasoactive substances, local activation of the renin-angiotensin system (RAS) is particularly important because it can lead to the constriction of efferent arterioles, hypoperfusion of postglomerular peritubular capillaries, and subsequent hypoxia of the tubulointerstitium in the downstream compartment. In addition, angiotensin II induces oxidative stress via the activation of NADPH oxidase. Oxidative stress damages endothelial cells directly, causing the loss of peritubular capillaries, and also results in relative hypoxia due to inefficient cellular respiration. Thus, angiotensin II induces renal hypoxia via both hemodynamic and nonhemodynamic mechanisms. In the past two decades, considerable gains have been realized in retarding the progression of chronic kidney disease by emphasizing blood pressure control and blockade of the RAS. Chronic hypoxia in the kidney is an ideal therapeutic target, and the beneficial effects of blockade of RAS in kidney disease are, at least in part, mediated by the amelioration of local hypoxia. (Hypertens Res 2008; 31: 175-184)
\end{abstract}

Key Words: ischemia, chronic kidney disease, angiotensin receptor blocker, oxidative stress

\section{Introduction}

While chronic kidney disease (CKD) was previously believed to be relatively uncommon, it is now recognized as a common public health problem of global concern (1). The most important adverse outcomes of CKD include not only the complications of decreased glomerular filtration rate (GFR) and progression to kidney failure, but also an increased risk of cardiovascular disease (2). Indeed, the strong association between CKD and cardiovascular disease has led the American Heart Association and National Kidney Foundation to recommend that all patients with cardiovascular disease be screened for evidence of kidney disease (3).

Primary insults differ among kidney diseases, including glomerulonephritis, diabetic nephropathy, and hypertensive nephrosclerosis. However, once renal damage reaches a certain threshold, progression of renal disease is consistent, irreversible, and largely independent of the initial insult. Functional impairment of the kidney correlates better with the degree of tubulointerstitial damage than with that of glomerular injury, and it is widely recognized that the final common pathway which mediates the deterioration of kidney failure is to be found in the tubulointerstitium (4).

From the ${ }^{1}$ Division of Nephrology and Endocrinology, University of Tokyo School of Medicine, Tokyo, Japan.

This work was supported in part by a Grant-in-Aid for Scientific Research from the Japan Society for the Promotion of Science (grant number 19390228). Address for Reprints: Masaomi Nangaku, M.D., Ph.D., Division of Nephrology and Endocrinology, University of Tokyo School of Medicine, 7-3-1 Hongo, Bunkyo-ku, Tokyo 113-8655, Japan. E-mail: mnangaku-tky@unin.ac.jp Received May 9, 2007; Accepted in revised form August 13, 2007. 


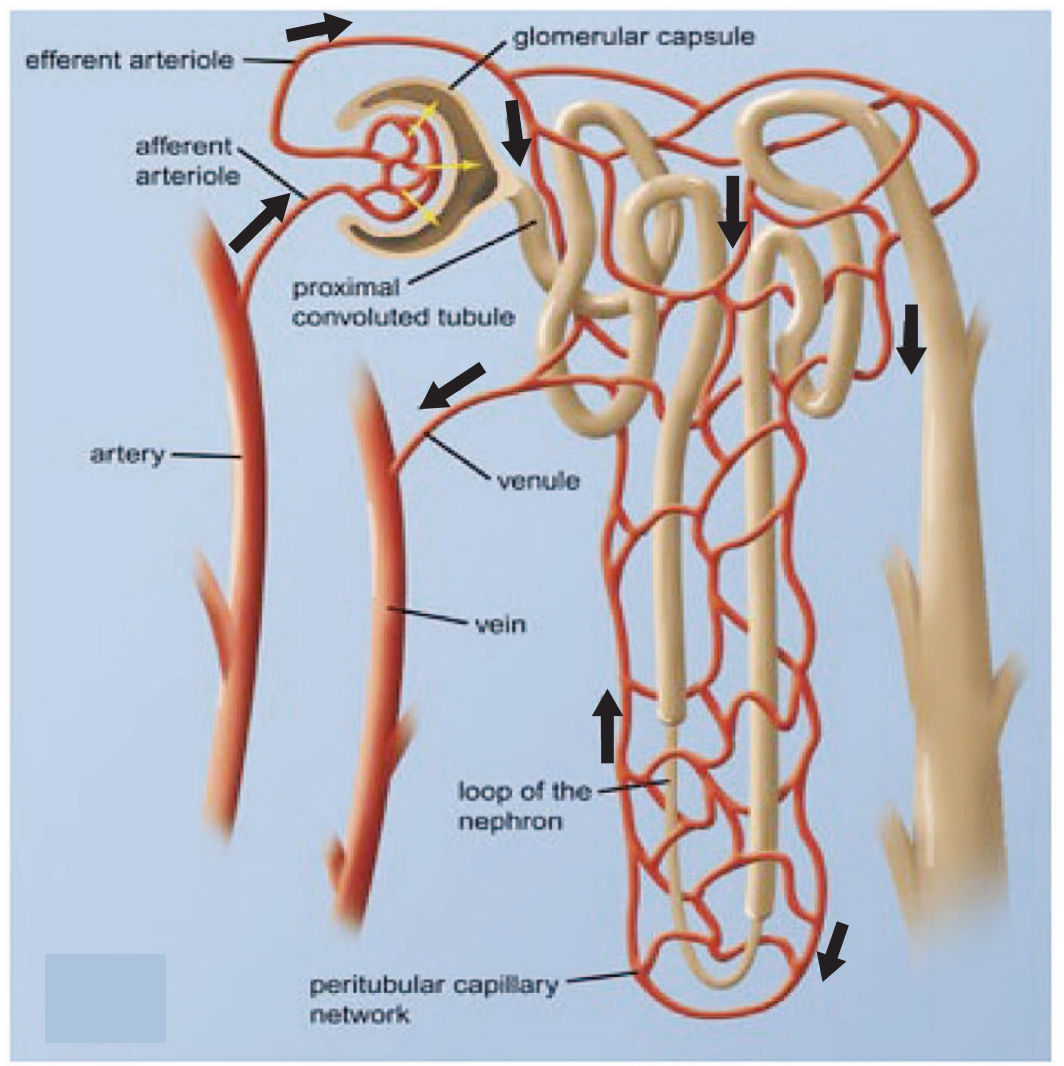

Fig. 1. The microvasculature of the nephron. The peritubular capillary plexus is fed by glomerular efferent arterioles and supplies oxygen to tubular and interstitial cells (modified from Nangaku (13)).

\section{Blockade of the Renin-Angiotensin System to Treat CKD}

While renal disease causes an increase in blood pressure (BP), high BP in turn accelerates the loss of function in the diseased kidney. The treatment of hypertension is thus now an important component in the treatment of CKD patients, not only to prevent cardiovascular complications but also to protect the kidney $(5,6)$. Meta-regression analyses have indicated that BP reduction accounts for $50 \%$ of the variance in GFR decline and that each $10-\mathrm{mmHg}$ reduction in mean arterial pressure (down to $92 \mathrm{mmHg}$ ) confers a benefit in GFR preservation of $3.7-5.0 \mathrm{~mL} / \mathrm{min}$ per year $(7-10)$. On this basis, stricter control of BP is recommended in patients with kidney injury.

Further, to maximize the protection of residual renal function, many clinical practice guidelines now suggest initial therapy with reagents to block the renin-angiotensin system (RAS). Why is blockade of RAS considered the gold standard in the treatment of patients with CKD? A large number of prospective, randomized, controlled studies have demonstrated the beneficial effects of angiotensin converting enzyme inhibitors (ACEIs) or angiotensin receptor blockers
(ARBs). These studies are covered in detail in our previous overview but, taken together, they ascribe the profound beneficial effects of blockade of RAS to reno-protection that goes beyond mere BP reduction (11). In particular, Weinberg et al. reported that the BP-lowering effects of an ARB, candesartan cilexetil (approved dosage range in Japan up to $12 \mathrm{mg}$ /day), reached a plateau when doses were increased to $96 \mathrm{mg} /$ day. In contrast, they observed dose-related reductions in urinary protein excretion without any further lowering of BP (12). While previous studies emphasized the amelioration of intraglomerular hypertension as a BP-independent reno-protective mechanism of blockade of RAS, more recent studies have clarified that the beneficial mechanism of ACEIs and ARBs includes a crucial role in ameliorating chronic hypoxia in the kidney.

\section{Microvasculature of the Kidney}

Although the kidneys receive a very high blood flow, oxygen extraction in the kidney is actually relatively low. This is due to its unique vasculature system, in which oxygen shunt diffusion occurs between arterial and venous vessels that run in close parallel contact in the kidney. Further, the maintenance of homeostasis mandates the reabsorption of a large fraction 
of the sodium and water filtered by the glomeruli. This reabsorption process is driven by active transport and uses a large amount of oxygen. As a consequence, the kidneys are particularly susceptible to hypoxic injury, and recent studies emphasize chronic hypoxia in the tubulointerstitium as a final common pathway to end-stage kidney disease (13-18).

In the kidney, afferent arterioles arise from the interlobular arteries. Except for branches that go toward the pelvic mucosa, all blood from the interlobular and arcuate arteries is directed into the glomerular capillary bed. The glomerular capillaries merge together again at the vascular pole to form the efferent arterioles. The glomerular efferent arterioles from the cortical glomeruli supply a fine capillary plexus which lies around the tubules beneath the capsule and in the areas of cortex between the interlobular vessels. The efferent vessels of the juxtamedullary glomeruli supply the subcortical capillary plexus, in addition to dividing into the vasa recta, which enter the medulla. The peritubular capillary plexus surrounds the tubules and supplies oxygen to tubular and interstitial cells (Fig. 1). Thus, whenever blood flow in the peritubular capillaries is impaired, the kidney suffers from hypoxia.

\section{Chronic Hypoxia of the Kidney: Animal Models}

In advanced kidney disease, peritubular capillary loss leads to hypoxia of the corresponding region. Evidence for this comes from a number of studies in a variety of experimental animal models demonstrating an association between peritubular capillary loss and the progression of renal injury (19-26).

Previously, we used intravital microscopy in a model of progressive glomerulonephritis induced by uni-nephrectomy and repeated injection of anti-Thy1 antibodies to measure peritubular capillary blood flow. The results showed the stagnation of peritubular capillary blood flow in the early phase of glomerulonephritis (27). Notably, this decrease in peritubular capillary blood flow occurred well before the development of structural capillary injury. Further, we also showed that the stagnation of peritubular blood flow was associated with hypoxia in the kidney, as demonstrated by the accumulation of pimonidazole, a reagent which binds to hypoxic cells.

Diabetic kidneys are also hypoxic. In a second study, we again used pimonidazole staining to demonstrate hypoxia in the kidneys of the spontaneously hypertensive rats (SHR)/ NDmc-cp rat, a model of type 2 diabetic nephropathy (28). Hypoxia of the diabetic kidney has also been demonstrated using the blood oxygen level-dependent (BOLD)-MRI technique (29): this method utilizes field distortion by deoxyhemoglobin in the magnetic field, which appears as BOLD contrast in the resulting images. The kidneys of streptozotocin-induced diabetic rats are hypoxic from an early stage (29).

\section{Hypoxia-Sensing Transgenic Animals}

Various methodological limitations have hampered the mea- surement of oxygen concentrations in vivo, and novel ways of monitoring oxygen in experimental animals have long been sought. All cells are endowed with a system which protects the cell against hypoxia, and this involves the hypoxia-inducible factor (HIF) and hypoxia-responsive element (HRE). In normoxia, the HIF- $\alpha$ subunit is hydroxylated, which enables the von Hipped-Lindau protein to bind to it, culminating in its degradation. Under hypoxic conditions, however, the HIF- $\alpha$ subunit escapes degradation, binding instead to the constitutively expressed HIF- $\beta$, and exerts its hypoxic response through binding to the cis-consensus HIF-binding site, namely the hypoxia-responsive element HRE. HIF regulates a variety of genes, such as erythropoietin (EPO) and vascular endothelial growth factor (VEGF).

We utilized this system to establish hypoxia-sensing transgenic rats expressing a luciferase reporter vector under the HRE-driven promoter. We used these rats to challenge the hypothesis that tubulointerstitial hypoxia occurs in the kidney during renal disease and modifies the pathogenic progression. In the puromycin nephrosis model, for example, hypoxic tubules were visualized diffusely in the cortex at both 1 and 2 weeks (30). In the remnant kidney model, on the other hand, hypoxic areas started to extend from the outer medulla to the cortex at week 1, and became more pronounced at week 4 . These rats also enabled us to reveal the age-related expansion of hypoxia in all areas of the kidney (31).

Safran et al. recently used a similar strategy to establish hypoxia-sensing transgenic mice (32). Using a photon-counting charge-coupled device camera to show bioluminescence after the administration of luciferin, they showed that the kidneys of these mice were already hypoxic under normoxic conditions. Further, when the mice were placed in a low-oxygen environment, various parts of the body showed a 5- to 10-fold increase in light emission, and hypoxia in the kidneys exceeded that in other organs.

\section{Activation of the RAS in Chronic Kidney Disease}

Activation of the RAS, which leads to an increase in the synthesis of angiotensin II, is a frequent finding in patients with CKD. In patients with CKD and associated volume overload, even a "normal" plasma concentration of renin and angiotensin II is in fact inappropriately high (33). Back in 1934, Goldblatt et al. demonstrated that the clamping of a renal artery produced hypertension in dogs (34), leading to the hypothesis that the kidney might release a pressor substance in response to ischemia, and eventually to our present understanding of the role of RAS in the regulation of BP (35). In patients with $\mathrm{CKD}$, the baroreceptors in the kidney which control the synthesis and secretion of renin are exposed to an inhomogeneous spectrum of perfusion pressures, because of the luminal narrowing of some, but not all, afferent preglomerular vessels. As a consequence, a proportion of glomeruli "senses" inappropriately low perfusion pressures and acti- 


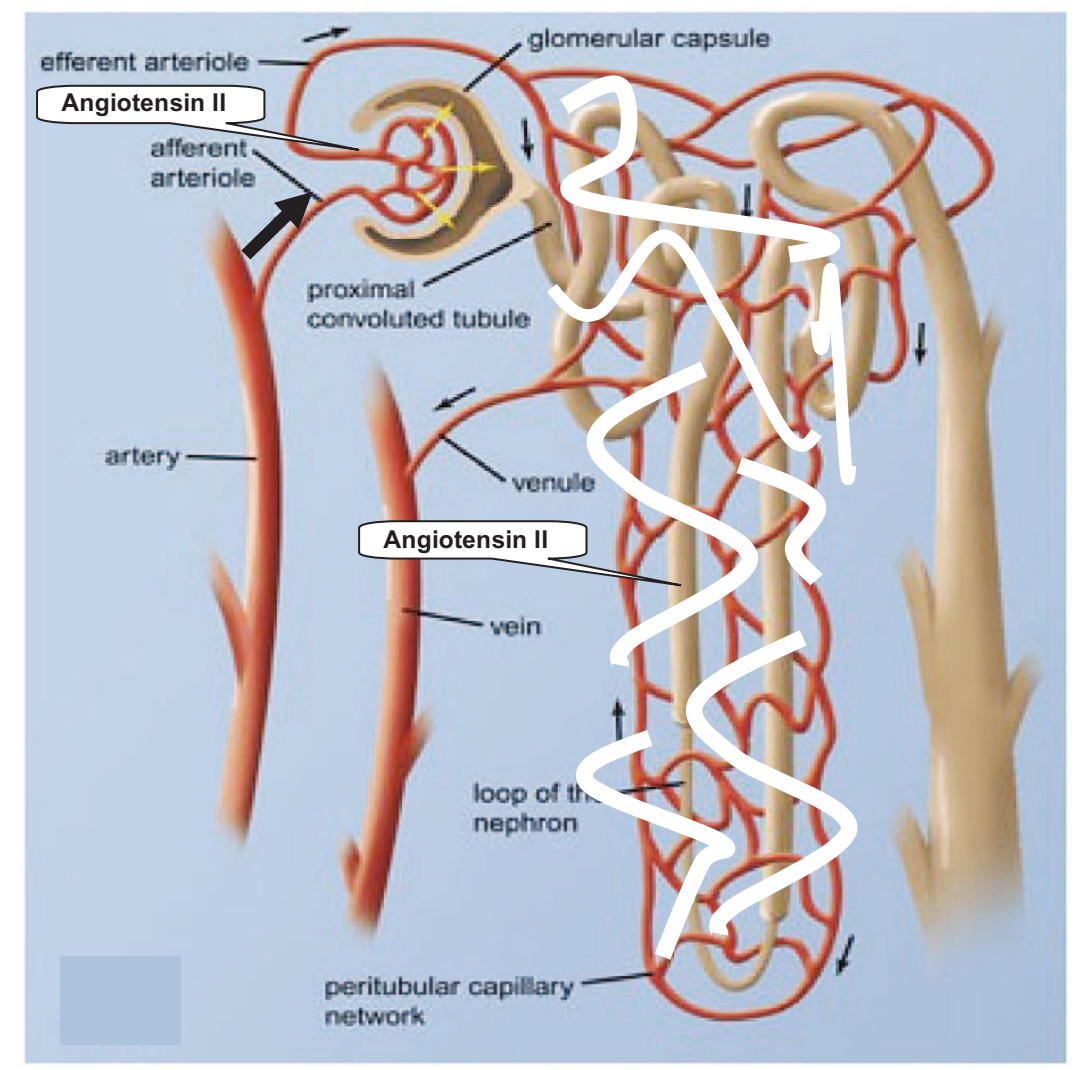

Fig. 2. Angiotensin II-induced chronic hypoxia in the kidney. Angiotensin II induces structural changes such as fibrosis and loss of peritubular capillaries. In addition, angiotensin II contributes to hypoxia of the kidney via functional changes, including stagnation of peritubular capillary blood flow and induction of oxidative stress (modified from Nangaku (13)).

vates RAS independently of the systemic BP.

Angiotensin II constricts precapillary arterioles and increases BP. Further, it stimulates aldosterone release from the adrenal cortex, which causes renal sodium retention and an increase in circulating blood volume. Of interest, angiotensin II levels in renal tissues are much higher than can be explained on the basis of equilibration with the circulating concentrations, and it is now apparent that activation of the local RAS plays a crucial role in kidney disease (36). Further, proteinuria, an established risk factor in the progression of renal disease, increases angiotensin II levels in tubular cells in a nuclear factor (NF)- $\mathrm{B}$-dependent manner (37).

In addition to its induction of systemic hypertension, angiotensin II plays a crucial role in chronic hypoxia of the kidney via structural and functional mechanisms, as described below.

\section{Angiotensin II-Induced Structural Changes in Renal Circulation}

Simply speaking, when there are no blood vessels, there is no blood flow. One of the major pathways of angiotensin IIinduced renal hypoxia is via structural changes in renal circulation. Angiotensin II damages the renal vasculature and induces fibrosis (Fig. 2). One way it does this is by stimulating the expression of a major fibrogenic cytokine, transforming growth factor (TGF)- $\beta$, in the kidney and upregulating receptors for TGF- $\beta$ (38). Fibrotic kidneys are devoid of peritubular capillary blood, and the corresponding region becomes hypoxic.

Among investigations of this effect, we previously demonstrated the loss of peritubular capillaries in angiotensin IIinfused rats by staining with an endothelium-specific antibody, JG-12 (39). This capillary loss was ameliorated by administration of an angiotensin receptor blocker, olmesartan. More recent studies utilizing another endotheliumspecific antibody, RECA-1, have confirmed the loss of peritubular capillaries in angiotensin II-infused rats (40). As in our study, the loss of peritubular capillaries and progression of fibrosis in these rats was also mitigated by an angiotensin receptor blocker.

With regard to fibrosis, the reduced efficiency of oxygen diffusion across the relatively extended distance between capillaries and tubular cells means that interstitial fibrosis impairs the tubular oxygen supply even in the presence of peritubular capillaries. A fibrogenic response induced by excessive angiotensin II in turn leads to the obliteration of 
peritubular capillaries and a decrease in blood supply. Subsequent hypoxia induces the transdifferentiation of tubular cells into myofibroblasts (epithelial mesenchymal transdifferentiation), predisposing the kidney to fibrosis (41). Hypoxia also induces the apoptosis of resident kidney cells (42-44). The overall effect is to induce a vicious cycle of fibrosis and regional hypoxia.

\section{Angiotensin II-Induced Functional Changes in Renal Circulation}

\section{Hemodynamic Changes}

In addition to its induction of structural changes, a second important mechanism by which angiotensin II induces renal hypoxia in kidney disorders is via functional changes at an early stage of renal disease, before the development of the structural changes. It does this by constricting efferent arterioles, thereby decreasing blood flow in post-glomerular peritubular capillaries (Fig. 2). Nishiyama's group recently provided direct evidence of the angiotensin II-induced reduction in blood flow by visualizing the superficial peritubular capillaries directly through an intravital fluorescence videomicroscope system (45). Evaluation of peritubular capillary blood flow by analyzing the velocity of fluoresceinlabeled erythrocytes showed a marked decrease in peritubular capillary blood flow in rats following the intravenous administration of angiotensin II.

Of note, hypoxia of the kidney by angiotensin II has also been demonstrated in humans. Schachinger et al. studied the effects of vasoactive agents on kidney oxygenation in humans utilizing BOLD-MRI (46). Angiotensin II caused an immediate shortening of $T_{2}{ }^{*}$, in contrast to other vasoactive agents which had no effect on the renal BOLD signal. The short onset latency of renal hypoxia induced by angiotensin II administration suggested that this response is a consequence of altered perfusion of the peritubular capillary plexus.

\section{Oxidative Stress}

In addition, angiotensin II induces hypoxia of the kidney via a second functional change, namely by NADPH oxidase activation-induced oxidative stress. Oxidative stress damages target organs via multiple mechanisms. For example, lectinlike oxidized low-density lipoprotein (LDL) receptor (LOX)1, a novel oxidized LDL (ox-LDL) receptor recently isolated from endothelial cells induced by a variety of stimuli, including angiotensin II $(47,48)$, acts as a functional receptor that mediates the cytotoxic effect of ox-LDL in various kidney disease models $(49,50)$.

Furthermore, oxidative stress alters oxygen metabolism and oxygen availability. Reactive oxygen species (ROS) react with nitric oxide (NO) to form peroxynitrite and thereby decrease the bioavailability of NO. NO controls mitochondrial respiration via the suppression of respiration under nor- mal conditions. A decrease in NO therefore results in the dysregulation of mitochondrial respiration, leading to inefficient oxygen usage. The potency of NO-mediated inhibition of renal oxygen usage was demonstrated by the pioneering study of Laycock et al., in which the administration of a nitric oxide synthase (NOS) inhibitor increased overall renal oxygen usage (51). The question of which isoform of NOS is responsible for the renal oxygen consumption remains controversial: while neuronal (n) NOS inhibition increases the oxygen costs of kidney function (52), studies utilizing mice deficient (-/-) in endothelial (e) NOS showed that regulation is due to NO production by eNOS (53).

Various experimental findings support the role of angiotensin II-induced oxidative stress in augmenting renal oxygen consumption and the subsequent induction of hypoxia. Welch et al. studied the early 2-kidney, 1-clip angiotensin IIdependent model. They demonstrated inefficient usage of oxygen in tubular transport in the clipped kidney. The reduced efficiency of renal oxygen usage was restored by the superoxide dismutase mimetic tempol (54). In addition, they recently extended these findings using rats which had received prolonged angiotensin II administration. Specifically, angiotensin II reduced the efficiency of renal oxygen usage in tubular sodium transport, resulting in a decrease in oxygen tension throughout the cortex. Tempol blunted all these effects of angiotensin II (55). Further, Adler and Huang showed that NO bioavailability in SHR is impaired due to an angiotensin II-mediated increase in superoxide production in association with enhanced expression of NADPH oxidase components (56). These results suggest that the oxidative stress induced by angiotensin II is associated with augmented oxygen consumption and subsequent hypoxia in the kidney.

The complexity of the relationship between hypoxia and oxidative stress is highlighted by the fact that, while oxidative stress aggravates hypoxia, the reverse also holds true: hypoxia aggravates oxidative stress. This may sound paradoxical, given that oxidative stress is a condition which requires oxygen and results in the excessive production of oxygen radicals beyond the antioxidant capacity. However, hypoxia per se stimulates xanthine oxidase and NADPH oxidase, resulting in the increased production of oxidative stress (57). The pathogenic role of oxidative stress under hypoxic conditions was further emphasized by studies on the pulmonary vasculature using adrenomedullin, a potent antioxidant $(58,59)$ that inhibits angiotensin II-induced oxidative stress (60). Chronic hypoxia was shown to induce pulmonary vascular remodeling, which was associated with an increased production of oxidative stress as measured by electron spin resonance and immunostaining of 3-nitrotyrosine. This pulmonary vascular remodeling was aggravated in heterozygous adrenomedullin-knockout mice, suggesting a pathogenic role of hypoxia and protection by endogenous adrenomedullin through the suppression of ROS generation (61). Thus, a vicious cycle of hypoxia and oxidative stress may participate in a variety of disorders, including CKD. 


\section{Angiotensin II and Podocyte Injury}

The evidence above emphasizes that the pathogenic role of the RAS in kidney failure operates via the induction of hypoxia in the tubulointerstitial compartment. However, the crucial role of glomerulosclerosis in the progression of kidney failure should not be ignored. The terminally differentiated podocyte, also called the glomerular visceral epithelial cell, is a highly specialized cell. An exciting and expanding body of evidence shows that, owing to the relative inability of these cells to proliferate, a reduction in podocyte number directly causes proteinuria and glomerulosclerosis $(62,63)$. While earlier studies have emphasized the role of glomerular hypertension hemodynamically induced by activation of the RAS in the development of glomerulosclerosis, more recent studies have highlighted the direct participation of angiotensin II in podocyte injury.

Among recent findings in regard to angiotensin II, ischemia of the rabbit kidney was shown to cause a flattening and spreading of major processes of podocytes, while in vitro incubation studies suggested that local angiotensin II may produce cell changes in the glomerular epithelium (64). Local expression of the RAS in podocytes was recently confirmed in human podocytes (65). Further, evidence of direct injury to podocytes was recently demonstrated in studies utilizing genetically engineered animals: a transgenic rat model with overexpression of the human angiotensin II type 1 receptor (hAT1) specifically in podocytes developed significant albuminuria without the development of hypertension. The glomerular damage in this model progressed to nephron loss via a well-known pathway typically seen in classic focal segmental glomerulosclerosis (66). Further, aldosterone, the final product of the angiotensin II-stimulated corticosteroid biosynthetic pathway, damages podocytes directly (67-69).

Thus, in addition to direct effects of the RAS on tubulointerstitial hypoxia, activation of this system can induce glomerulosclerosis and anatomical damage to glomerular tufts, with a subsequent decrease in post-glomerular capillary perfusion in association with hypoxia in the tubulointerstitial compartment.

\section{HIF-Activating Therapy against Renal Hypoxia}

Chronic hypoxia in the kidney is the final common pathway to end-stage renal disease. Therapeutic approaches targeting hypoxia in the kidney should therefore be effective in patients with CKD. One promising approach to protecting tissues against hypoxia might be activation of a "master gene" switch, HIF.

Our recent studies revealed a postnatal biological role of HIF-2 $\alpha$ in the kidney (70). We induced the well-established ischemia reperfusion injury model of the kidney in HIF- $2 \alpha$ knockdown $(\mathrm{kd})$ mice. Although ischemia impaired renal

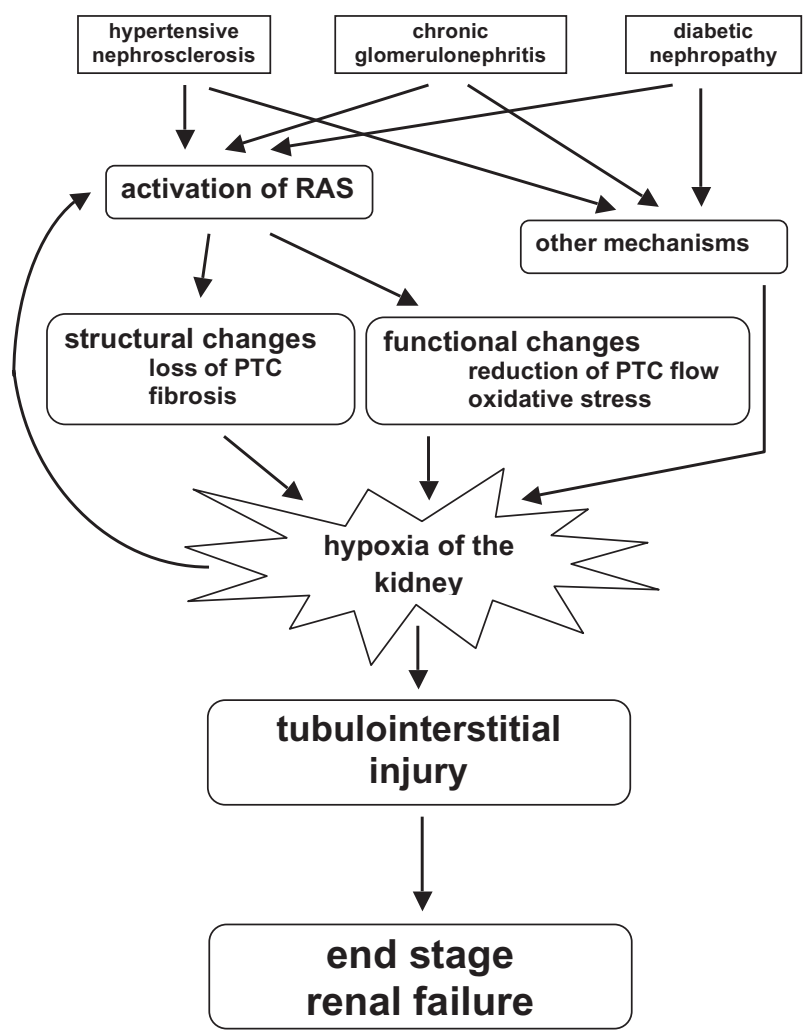

Fig. 3. Schematic view of hypoxia-induced tubulointerstitial injury as a final common pathway to end-stage renal failure Other mechanisms of chronic hypoxia of the kidney include decreased renal blood flow as a result of atherosclerosis, increased metabolic demands of tubular cells due to glomerular hyperfiltration, and decreased oxygen delivery as a result of renal anemia. A decrease in renal blood flow due to activation of the RAS leads to stimulation of the macula densa and subsequent secretion of renin, instituting a vicious cycle of hypoxia and RAS activation. PTC, peritubular capillary.

function in both wild-type and HIF-2 $\alpha$ kd mice, HIF- $2 \alpha \mathrm{kd}$ mice had significantly higher blood urea nitrogen (BUN) levels and more severe histological changes. Evaluation of oxidative stress markers showed greater oxidative stress in the HIF- $2 \alpha \mathrm{kd}$ mice, while examination of the expression of antioxidative enzyme genes in these mice showed that the expression of SOD1, SOD2 and GPX1 genes was significantly lower in the HIF-2 $\alpha$ kd kidney. In contrast, expression of HO1 , a target gene of HIF-1 $\alpha$, did not differ between the wildtype and HIF- $2 \alpha$ kd kidneys. Judging by the localization of HIF- $2 \alpha$ in the kidney, we speculated that HIF- $2 \alpha$ in the kidney endothelium is responsible for the regulation of oxidative stress. To examine this, knockdown of the HIF- $2 \alpha$ gene was achieved by insertion of the neomycin gene sandwiched between two loxP sequences. When the expression of HIF-2 $\alpha$ in the endothelium was specifically restored by intercrossing 
HIF- $2 \alpha$ kd with Tie1-Cre mice, the susceptibility of HIF- $2 \alpha$ $\mathrm{kd}$ mice to renal ischemia was found to be restored in the resulting HIF-2 $\alpha \mathrm{kd} /$ Tie1-Cre mice. This result clearly demonstrated a protective role of HIF- $2 \alpha$ in the endothelium, and raised the possibility that the stimulation of HIF may be a powerful tool in protecting the vasculature under hypoxic conditions.

In support of this notion, gene transfer of constitutively active HIF (HIF/VP16) was found to induce the expression of various HIF-regulated genes and to protect the medulla against ischemic insults in rats (71). Further, a recent phase I dose-escalation study on the adenoviral delivery of a constitutively active form of transcription factor HIF-1 $\alpha$ (Ad2/HIF$1 \alpha / \mathrm{VP} 16)$ into the lower extremity of patients with critical limb ischemia showed that HIF-1 $\alpha$ therapy in patients with critical limb ischemia was well tolerated. Of note, no amputations occurred in the two highest-dose groups of Ad2/HIF$1 \alpha /$ VP16 (72).

Previous studies by our group and others have demonstrated that stimulation of HIF with cobalt chloride is effective in a variety of kidney disease models (73-77). Furthermore, pretreatment with either carbon monoxide, leading to tissue hypoxia, or the novel prolyl hydroxylase inhibitor FG-4487 strongly induced the accumulation of HIF$1 \alpha$ and HIF- $2 \alpha$ in tubular and peritubular cells, respectively, with significant amelioration of ischemic renal injury (78).

\section{Angiotensin Blockade as a Therapeutic Modality against Renal Hypoxia}

Although HIF stimulation holds promise as a future therapy, at present the best modality for the treatment of kidney disease is blockade of the RAS (11). One important mechanism of the BP-independent renoprotective effect of this blockade is the preservation of peritubular capillary perfusion.

Norman et al. investigated the effects of RAS blockade on renal oxygenation in anesthetized adult rats (79). Cortical microvascular oxygenation on the surface of the exposed kidney was measured utilizing the porphyrin phosphorescence technique. The results showed a slow decline in cortical oxygenation in control animals over the 3-h experimental period. Administration of an ACEI or ARB at the beginning of the experimental period completely abrogated this decline.

Further, we demonstrated a decrease in blood flow in peritubular capillaries and subsequent hypoxia in a very early phase of remnant kidneys (week 1) (80). These changes were associated with the narrowing and distortion of peritubular capillaries, but not with a decrease in the number of peritubular capillaries. The physiologic perfusion status of the peritubular capillary network was evaluated by the lectin perfusion and Hoechst dye diffusion techniques. Treatment of these animals with an ARB restored the blood flow in peritubular capillaries and improved the oxygenation of the kidney.

Long-term administration of an ARB in type 2 diabetic rats also resulted in the restoration of oxygenation in the kidney
(28). We and others have demonstrated the anti-oxidative stress effects of ARBs. Because angiotensin II induces oxidative stress via the activation of NADPH oxidase, as described above, blockade of the receptor inhibits this stress. Furthermore, the chemical structures of ARBs inhibit in vitro oxidative stress by chelating transition metals and inhibiting various oxidative steps in a receptor-independent manner $(81$, 82 ). Thus, the mechanisms of the improvement in oxygenation by renin-angiotensin blockade include both hemodynamic changes via the dilatation of glomerular efferent arterioles and efficient oxygen usage via the amelioration of oxidative stress. Supporting this notion, Welch et al. demonstrated that the administration of an ARB improved the inefficient utilization of oxygen in sodium transport in the SHR kidney (83).

\section{Conclusion}

Chronic hypoxia in kidney disease serves as the final common pathway leading to end-stage renal failure (Fig. 3). Angiotensin II causes hypoxia in the kidney by inducing structural microvasculature damage and fibrotic changes. It also induces the constriction of glomerular efferent arterioles, resulting in a reduction in peritubular capillary blood flow and subsequent hypoxia in the corresponding region. Further, angiotensin II induces oxidative stress, which in turn consumes nitric oxide and results in inefficient oxygen usage. Therapeutic approaches against this final common pathway utilizing an ACEI or ARB are effective in a broad range of renal diseases.

\section{References}

1. Kiberd B: The chronic kidney disease epidemic: stepping back and looking forward. J Am Soc Nephrol 2006; 17: 2967-2973.

2. Sarnak MJ, Levey AS, Schoolwerth AC, et al: Kidney disease as a risk factor for development of cardiovascular disease: a statement from the American Heart Association Councils on Kidney in Cardiovascular Disease, High Blood Pressure Research, Clinical Cardiology, and Epidemiology and Prevention. Hypertension 2003; 42: 1050-1065.

3. Brosius FC 3rd, Hostetter TH, Kelepouris E, et al: Detection of chronic kidney disease in patients with or at increased risk of cardiovascular disease: a science advisory from the American Heart Association Kidney and Cardiovascular Disease Council; the Councils on High Blood Pressure Research, Cardiovascular Disease in the Young, and Epidemiology and Prevention; and the Quality of Care and Outcomes Research Interdisciplinary Working Group: Developed in Collaboration with the National Kidney Foundation. Hypertension 2006; 48: 751-755.

4. Nangaku M, Fujita T: Chronic interstitial nephritis, in Johnson RJ, Feehally J, Floege J (eds): Comprehensive Clinical Nephrology, 3rd ed. Philadelphia, Elsevier, 2007, pp 703-716.

5. Flack JM, Peters R, Shafi T, Alrefai H, Nasser SA, Crook 
E: Prevention of hypertension and its complications: theoretical basis and guidelines for treatment. J Am Soc Nephrol 2003; 14 (Suppl 2): S92-S98.

6. Levey AS: Nondiabetic kidney disease. N Engl J Med 2002; 347: 1505-1511.

7. Bakris GL, Weir MR: Angiotensin-converting enzyme inhibitor associated elevations in serum creatinine: is this a cause for concern? Arch Intern Med 2000; 160: 685-693.

8. Maki DD, Ma JZ, Louis TA, Kasiske BL: Long-term effects of antihypertensive agents on proteinuria and renal function. Arch Intern Med 1995; 155: 1073-1080.

9. Kasiske BL, Kalil RS, Ma JZ, Liao M, Keane WF: Effect of antihypertensive therapy on the kidney in patients with diabetes: a meta-regression analysis. Ann Intern Med 1993; 118: $129-138$.

10. Jafar TH, Schmid CH, Landa M, et al: Angiotensin-converting enzyme inhibitors and progression of nondiabetic renal disease. A meta-analysis of patient-level data. Ann Intern Med 2001; 135: 73-87.

11. Nangaku M, Ohse T, Tanaka T, Kojima I, Fujita T: Renoprotection with anti-hypertensives: reduction of proteinuria and improvement of oxygenation via inhibition of the reninangiotensin system. Curr Hypertens Rev 2005; 1: 67-76.

12. Weinberg MS, Weinberg AJ, Cord R, Zappe DH: The effect of high dose angiotensin II receptor blockade beyond maximal recommended doses in reducing urinary protein excretion. J Renin Angiotensin Aldosterone Syst 2001; 2 (Suppl 1): S196-S198.

13. Nangaku M: Chronic hypoxia and tubulointerstitial injury: a final common pathway to end-stage renal failure. $J \mathrm{Am} \mathrm{Soc}$ Nephrol 2006; 17: 17-25.

14. Norman JT, Fine LG: Intrarenal oxygenation in chronic renal failure. Clin Exp Pharmacol Physiol 2006; 33: 989996.

15. Eckardt KU, Bernhardt WM, Weidemann A, et al: Role of hypoxia in the pathogenesis of renal disease. Kidney Int 2005; 99: S46-S51.

16. Nakagawa T, Kang DH, Ohashi R, et al: Tubulointerstitial disease: role of ischemia and microvascular disease. Curr Opin Nephrol Hypertens 2003; 12: 233-241.

17. Nangaku M: Hypoxia and tubulointerstitial injury: a final common pathway to end-stage renal failure. Nephron Exp Nephrol 2004; 98: e8-e12.

18. Nangaku M: Mechanisms of tubulointerstitial injury in the kidney: final common pathways to end-stage renal failure. Intern Med 2004; 43: 9-17.

19. Ohashi R, Shimizu A, Masuda Y, et al: Peritubular capillary regression during the progression of experimental obstructive nephropathy. J Am Soc Nephrol 2002; 13: 1795-1805.

20. Ohashi R, Kitamura H, Yamanaka N: Peritubular capillary injury during the progression of experimental glomerulonephritis in rats. J Am Soc Nephrol 2000; 11: 47-56.

21. Kang DH, Anderson S, Kim YG, et al: Impaired angiogenesis in the aging kidney: vascular endothelial growth factor and thrombospondin-1 in renal disease. Am J Kidney Dis 2001; 37: 601-611.

22. Kang DH, Hughes J, Mazzali M, Schreiner GF, Johnson RJ: Impaired angiogenesis in the remnant kidney model: II. Vascular endothelial growth factor administration reduces renal fibrosis and stabilizes renal function. $\mathrm{J} \mathrm{Am} \mathrm{Soc} \mathrm{Neph-}$ rol 2001; 12: 1448-1457.

23. Kairaitis LK, Wang Y, Gassmann M, Tay YC, Harris DC: HIF-1alpha expression follows microvascular loss in advanced murine adriamycin nephrosis. Am J Physiol Renal Physiol 2005; 288: F198-F206.

24. Yuan HT, Li XZ, Pitera JE, Long DA, Woolf AS: Peritubular capillary loss after mouse acute nephrotoxicity correlates with down-regulation of vascular endothelial growth factorA and hypoxia-inducible factor-1 alpha. Am J Pathol 2003; 163: 2289-2301.

25. Sun D, Feng J, Dai C, et al: Role of peritubular capillary loss and hypoxia in progressive tubulointerstitial fibrosis in a rat model of aristolochic acid nephropathy. Am J Nephrol 2006; 26: 363-371.

26. Zhang B, Liang X, Shi W, et al: Role of impaired peritubular capillary and hypoxia in progressive interstitial fibrosis after 56 subtotal nephrectomy of rats. Nephrology 2005; 10: 351-357.

27. Matsumoto M, Tanaka T, Yamamoto T, et al: Hypoperfusion of peritubular capillaries induces chronic hypoxia before progression of tubulointerstitial injury in a progressive model of rat glomerulonephritis. J Am Soc Nephrol 2004; 15: 1574-1581.

28. Izuhara $\mathrm{Y}$, Nangaku M, Inagi $\mathrm{R}$, et al: Renoprotective properties of angiotensin receptor blockers beyond blood pressure lowering. J Am Soc Nephrol 2005; 16: 3631-3641.

29. Ries M, Basseau F, Tyndal B, et al: Renal diffusion and BOLD MRI in experimental diabetic nephropathy. J Magn Reson Imaging 2003; 17: 104-113.

30. Tanaka $T$, Miyata $T$, Inagi $R$, Fujita $T$, Nangaku M: Hypoxia in renal disease with proteinuria and/or glomerular hypertension. Am J Pathol 2004; 165: 1979-1992.

31. Tanaka T, Kato H, Kojima I, et al: Hypoxia and expression of hypoxia-inducible factor in the aging kidney. $J$ Gerontol A Biol Sci Med Sci 2006; 61: 795-805.

32. Safran M, Kim WY, O'Connell F, et al: Mouse model for noninvasive imaging of HIF prolyl hydroxylase activity: assessment of an oral agent that stimulates erythropoietin production. Proc Natl Acad Sci U S A 2006; 103: 105-110.

33. Lazarus JM, Hampers CL, Merrill JP: Hypertension in chronic renal failure: treatment with hemodialysis and nephrectomy. Arch Intern Med 1974; 133: 1059-1065.

34. Goldblatt H, Lynch J, Hanzal RF, Summerville WW: Studies on experimental hypertension: Part I: the production of persistent elevation of systolic blood pressure by means of renal ischemia. J Exp Med 1934; 59: 347-379.

35. Oparil S, Haber E: The renin-angiotensin system. $N$ Engl J Med 1974; 291: 389-401.

36. Kobori H, Nangaku M, Navar LG, Nishiyama A: Independent regulation of intrarenal angiotensin II and impact of antihypertensive agents. Pharmacol Rev 2007; 59: 251287.

37. Takase O, Marumo T, Imai N, et al: NF-kappaB-dependent increase in intrarenal angiotensin II induced by proteinuria. Kidney Int 2005; 68: 464-473.

38. Wolf G: Renal injury due to renin-angiotensin-aldosterone system activation of the transforming growth factor-beta pathway. Kidney Int 2006; 70: 1914-1919.

39. Shao J, Nangaku M, Miyata T, et al: Imbalance of T-cell subsets in angiotensin II-infused hypertensive rats with kid- 
ney injury. Hypertension 2003; 42: 31-38.

40. Kitayama H, Maeshima Y, Takazawa Y, et al: Regulation of angiogenic factors in angiotensin II infusion model in association with tubulointerstitial injuries. Am J Hypertens 2006; 19: 718-727.

41. Manotham K, Tanaka T, Matsumoto M, et al: Transdifferentiation of cultured tubular cells induced by hypoxia. Kidney Int 2004; 65: 871-880.

42. Tanaka T, Nangaku M, Miyata T, et al: Blockade of calcium influx through L-type calcium channels attenuates mitochondrial injury and apoptosis in hypoxic renal tubular cells. J Am Soc Nephrol 2004; 15: 2320-2333.

43. Tanaka T, Miyata T, Inagi R, et al: Hypoxia-induced apoptosis in cultured glomerular endothelial cells - involvement of mitochondrial pathways. Kidney Int 2003; 64: 20202032.

44. Tanaka T, Hanafusa N, Ingelfinger JR, Ohse T, Fujita T, Nangaku M: Hypoxia induces apoptosis in SV40-immortalized rat proximal tubular cells through the mitochondrial pathways, devoid of HIF-1-mediated upregulation of Bax. Biochem Biophys Res Commun 2003; 309: 222-231.

45. Kondo N, Kiyomoto H, Yamamoto T, et al: Effects of calcium channel blockade on angiotensin II-induced peritubular ischemia in rats. $J$ Pharmacol Exp Ther 2006; 316: 1047-1052.

46. Schachinger H, Klarhofer M, Linder L, Drewe J, Scheffler $\mathrm{K}$ : Angiotensin II decreases the renal MRI blood oxygenation level-dependent signal. Hypertension 2006; 47: 1062-1066.

47. Ando K, Fujita T: Role of lectin-like oxidized low-density lipoprotein receptor-1 (LOX-1) in the development of hypertensive organ damage. Clin Exp Nephrol 2004; 8: 178-182.

48. Nagase M, Ando K, Nagase T, Kaname S, Sawamura T, Fujita T: Redox-sensitive regulation of LOX-1 gene expression in vascular endothelium. Biochem Biophys Res Commun 2001; 281: 720-725.

49. Ueno T, Kaname S, Takaichi K, et al: LOX-1, an oxidized low-density lipoprotein receptor, was upregulated in the kidneys of chronic renal failure rats. Hypertens Res 2003; 26: $117-122$.

50. Nagase M, Kaname S, Nagase T, et al: Expression of LOX1 , an oxidized low-density lipoprotein receptor, in experimental hypertensive glomerulosclerosis. J Am Soc Nephrol 2000; 11: 1826-1836.

51. Laycock SK, Vogel T, Forfia PR, et al: Role of nitric oxide in the control of renal oxygen consumption and the regulation of chemical work in the kidney. Circ Res 1998; 82: 1263-1271.

52. Deng A, Miracle CM, Suarez JM, et al: Oxygen consumption in the kidney: effects of nitric oxide synthase isoforms and angiotensin II. Kidney Int 2005; 68: 723-730.

53. Adler $\mathrm{S}$, Huang $\mathrm{H}$, Loke KE, et al: Endothelial nitric oxide synthase plays an essential role in regulation of renal oxygen consumption by NO. Am J Physiol Renal Physiol 2001; 280: F838-F843.

54. Welch WJ, Mendonca M, Aslam S, Wilcox CS: Roles of oxidative stress and AT1 receptors in renal hemodynamics and oxygenation in the postclipped $2 \mathrm{~K}, 1 \mathrm{C}$ kidney. Hypertension 2003; 41: 692-696.
55. Welch WJ, Blau J, Xie H, Chabrashvili T, Wilcox CS: Angiotensin-induced defects in renal oxygenation: role of oxidative stress. Am J Physiol Heart Circ Physiol 2005; 288: $\mathrm{H} 22-\mathrm{H} 28$.

56. Adler S, Huang H: Oxidant stress in kidneys of spontaneously hypertensive rats involves both oxidase overexpression and loss of extracellular superoxide dismutase. Am J Physiol Renal Physiol 2004; 287: F907-F913.

57. Abramov AY, Scorziello A, Duchen MR: Three distinct mechanisms generate oxygen free radicals in neurons and contribute to cell death during anoxia and reoxygenation. $J$ Neurosci 2007; 27: 1129-1138.

58. Shimosawa T, Fujita T: Adrenomedullin and its related peptide. Endocr J 2005; 52: 1-10.

59. Ando K, Shimosawa T, Fujita T: Adrenomedullin in vascular diseases. Curr Hypertens Rep 2004; 6: 55-59.

60. Liu J, Shimosawa T, Matsui H, et al: Adrenomedullin inhibits angiotensin II-induced oxidative stress via Cskmediated inhibition of Src activity. Am J Physiol Heart Circ Physiol 2007; 292: H1714-H1721.

61. Matsui H, Shimosawa T, Itakura K, Guanqun X, Ando K, Fujita T: Adrenomedullin can protect against pulmonary vascular remodeling induced by hypoxia. Circulation 2004; 109: 2246-2251.

62. Shankland SJ: The podocyte's response to injury: role in proteinuria and glomerulosclerosis. Kidney Int 2006; 69: 2131-2147.

63. Kriz W, Lemley KV: The role of the podocyte in glomerulosclerosis. Curr Opin Nephrol Hypertens 1999; 8: 489497.

64. Racusen LC, Prozialeck DH, Solez K: Glomerular epithelial cell changes after ischemia or dehydration. Possible role of angiotensin II. Am J Pathol 1984; 114: 157-163.

65. Liebau MC, Lang D, Bohm J, et al: Functional expression of the renin-angiotensin system in human podocytes. Am J Physiol Renal Physiol 2006; 290: F710-F719.

66. Hoffmann S, Podlich D, Hahnel B, Kriz W, Gretz N: Angiotensin II type 1 receptor overexpression in podocytes induces glomerulosclerosis in transgenic rats. $J \mathrm{Am}$ Soc Nephrol 2004; 15: 1475-1487.

67. Shibata S, Nagase M, Yoshida S, Kawachi H, Fujita T: Podocyte as the target for aldosterone: roles of oxidative stress and Sgk1. Hypertension 2007; 49: 355-364.

68. Nagase M, Yoshida S, Shibata S, et al: Enhanced aldosterone signaling in the early nephropathy of rats with metabolic syndrome: possible contribution of fat-derived factors. J Am Soc Nephrol 2006; 17: 3438-3446.

69. Nagase M, Shibata S, Yoshida S, Nagase T, Gotoda T, Fujita T: Podocyte injury underlies the glomerulopathy of Dahl salt-hypertensive rats and is reversed by aldosterone blocker. Hypertension 2006; 47: 1084-1093.

70. Kojima I, Tanaka T, Inagi R, et al: Protective role of HIF-2 alpha against ischemic damage and oxidative stress in the kidney. J Am Soc Nephrol 2007; 18: 1218-1226.

71. Manotham K, Tanaka T, Ohse T, et al: A biological role of HIF-1 in the renal medulla. Kidney Int 2005; 67: 14281439.

72. Rajagopalan S, Olin J, Deitcher S, et al: Use of a constitutively active hypoxia-inducible factor-1alpha transgene as a therapeutic strategy in no-option critical limb ischemia 
patients: phase I dose-escalation experience. Circulation 2007; 115: 1234-1243.

73. Tanaka T, Matsumoto M, Inagi R, et al: Induction of protective genes by cobalt ameliorates tubulointerstitial injury in the progressive Thy1 nephritis. Kidney Int 2005; 68: 2714-2725.

74. Tanaka T, Kojima I, Ohse T, et al: Cobalt promotes angiogenesis via hypoxia-inducible factor and protects tubulointerstitium in the remnant kidney model. Lab Invest 2005; 85: 292-1307.

75. Tanaka T, Kojima I, Ohse T, et al: Hypoxia-inducible factor modulates tubular cell survival in cisplatin nephrotoxicity. Am J Physiol Renal Physiol 2005; 289: F1123-F1133.

76. Kudo Y, Kakinuma Y, Mori Y, et al: Hypoxia-inducible factor-1alpha is involved in the attenuation of experimentally induced rat glomerulonephritis. Nephron Exp Nephrol 2005; 100: e95-e103.

77. Matsumoto M, Makino Y, Tanaka T, et al: Induction of renoprotective gene expression by cobalt ameliorates ischemic injury of the kidney in rats. $J$ Am Soc Nephrol 2003; 14: 1825-1832.

78. Bernhardt WM, Campean V, Kany S, et al: Preconditional activation of hypoxia-inducible factors ameliorates ischemic acute renal failure. J Am Soc Nephrol 2006; 17: 1970-1978.

79. Norman JT, Stidwill R, Singer M, Fine LG: Angiotensin II blockade augments renal cortical microvascular $p \mathrm{O}_{2}$ indicating a novel, potentially renoprotective action. Nephron Physiol 2003; 94: 39-46.

80. Manotham K, Tanaka T, Matsumoto M, et al: Evidence of tubular hypoxia in the early phase in the remnant kidney model. J Am Soc Nephrol 2004; 15: 1277-1288.

81. Miyata T, van Ypersele de Strihou C, Ueda Y, et al: Angiotensin II receptor antagonists and angiotensin-converting enzyme inhibitors lower in vitro the formation of advanced glycation end products: biochemical mechanisms. J Am Soc Nephrol 2002; 13: 2478-2487.

82. Shao J, Nangaku M, Inagi R, et al: Receptor-independent intracellular radical scavenging activity of an angiotensin II receptor blocker. J Hypertens 2007; 25: 1643-1649.

83. Welch WJ, Baumgartl H, Lubbers D, Wilcox CS: Renal oxygenation defects in the spontaneously hypertensive rat: role of AT1 receptors. Kidney Int 2003; 63: 202-208. 